\title{
Successful management of nose arterial occlusion and impending skin necrosis after filler injection
}

\author{
Lenzo Ing Heong Ling, MB BCh BAO, FKCCS (1) \\ GEM Clinic Group, Kuala Lumpur, Malaysia
}

The growing use of dermal fillers, specifically the use of hyaluronic acid, may be explained by their effectiveness and versatility and their favorable safety profiles. Nevertheless, early and late complications with varying levels of severity may occur. The incidence of complications is low; however, more severe events such as skin ischemia and necrosis, and even visual loss may occur. The main objective of this case report on intravascular filler injection was to provide information on early signs of ischemic skin necrosis to physicians administering cosmetic fillers. This knowledge will facilitate timely management of symptoms, and enable treatment with hyperbaric oxygen therapy (HBOT) to prevent permanent scarring. A case note study of a single 41-year-old female who underwent a combination of treatments including the "high-dose pulsed hyaluronidase" technique, intravenous dexamethasone, HBOT, open dressing with normal saline, and bactigras dressing. Early recognition and immediate application of the correct combination of treatments for intravascular filler injury prevented further skin necrosis, permanent scarring, and the requirement for surgical reconstruction. Recently, as the application of dermal fillers for soft-tissue augmentation has increased, the number of cases reporting adverse reactions has also increased. The most severe and alarming early complication is vascular occlusion. Thus, it is crucial that physicians are familiar with the anatomy of the treated region, type of product used, correct application technique, and possible adverse events to allow immediate recognition and treatment.

Keywords: arterial embolization; dermal filler injection; hyperbaric oxygen therapy; skin necrosis; vascular compromise

\section{Introduction}

For the last three decades, dermal fillers have been injected with increasing frequency for soft-tissue augmentation by volume expansion in the management of the aging face [1]. The substances used as fillers include hyaluronic acid (HA) products, collagen, paraffin, and liquid silicone. However, recent safety concerns have reduced the acceptable materials, and over the last decade, HA, calcium hydroxyapatite (CaHA), polycaprolactone (PCL), polymethylmethacrylate beads (PMMA), and fat have been deemed safe and effective for facial rejuvenation, with immediate aesthetic effects.
The most widely used absorbable filler is HA due to its potential reversibility with hyaluronidase, an advantage in cases of patient dissatisfaction or possible adverse events [2,3].

Although HA is considered very safe, early and late complications with varying levels of severity may occur. Nevertheless, the incidence of complications is low, and the majority of adverse events are mild (edema, erythema, local ecchymosis, and nodule formation) and of limited duration. However, more severe events, such as ischemia and necrosis, may occur [4-6].

Vascular compromise is one of the most devastating complications arising from facial filler injections, either as inadvertent intra-arterial embolization or vascular compression, leading to

Received November 20, 2019; Revised December 20, 2019; Accepted December 20, 2019

Corresponding author: Lenzo Ing Heong Ling

E-mail:drlenzolih@gemclinic.com.my

This is an Open Access article distributed under the terms of the Creative Commons Attribution Non-Commercial License (http://creativecommons.org/licenses/by-nc/4.0), which permits unrestricted non-commercial use, distribution, and reproduction in any medium, provided the original work is properly cited.

Copyright @ 2019 Korean Society of Korean Cosmetic Surgery and Medicine (KSKCS \& KCCS). 
localized impending skin necrosis, skin necrosis, or even vision loss. This case report aimed to emphasize the importance of early detection and intervention of vascular compromise after filler injection, preventing development of necrosis and possible permanent scarring, and avoiding the necessity for reconstructive surgery $[7,8]$.

\section{Case report}

A 41-year-old female was injected over her nose area by an experienced injector with $>8$ years of practice. The injections were performed with a $27 \mathrm{G}$ sharp needle, and an HA filler concentration of $20 \mathrm{mg} / \mathrm{ml}$ plus lignocaine. In a single injection session, a total of $0.75 \mathrm{ml}$ was injected along the nasal bridge.

Pre-filler injection, no local anesthesia was used, and the patient complained of slight pain and discomfort during and after the injection. Subsequently, pain and discomfort persisted, and worsened over the next few hours after the patient went home. A split second of blanching was noticed by the clinician during the injection.

Fifteen hours after the filler injection, the patient presented with worsening pain and discomfort, and a clinical appearance of dusky blue-grey-black skin discoloration or livedo reticularis pattern was noted (Fig. 1), with the nose tip swollen and extending up to the nose radix. The involved area corresponded with the supply region for the dorsal nasal artery, and its communication with the lateral nasal artery. Capillary refill time (CRT) was slow in this area.

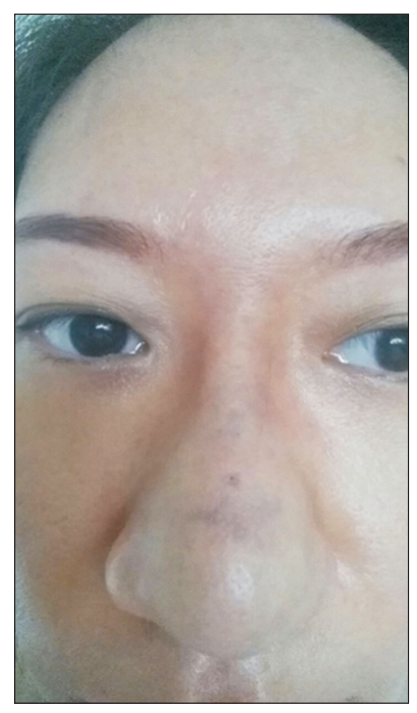

Fig. 1. A lesion of vascular compromise on the nasal tip extending to the nasal bridge. Dusky blue-grey-black skin discoloration, with swollen nose tip extending to nasal ridge region.
The decision was made to dissolve the HA filler material using the "high-dose pulsed hyaluronidase" technique. Because mainly the nose was involved, $500 \mathrm{U}$ of hyaluronidase, as per the guidelines, were injected with a $30 \mathrm{G}$ needle into the affected areas. Multiple injections were administered to cover the area and extended an additional $1 \mathrm{~cm}$ beyond the area of livedo reticularis. Concurrently, an intravenous (IV) dexamethasone 8 mg stat dose was administered to reduce any further inflammatory processes while awaiting return of reperfusion.

Within 1 minute, reperfusion with slightly improved CRT was noted in the involved area. After another 30 minutes, mottling was still present in most portions of affected areas, and a further $500 \mathrm{U}$ of hyaluronidase was injected in the dorsum of the nose with a $30 \mathrm{G}$ needle. Reperfusion in the remaining areas was noted, and the patient was injected a third time with $500 \mathrm{U}$ of hyaluronidase after another 30 minutes. Subsequently, the patient was observed for 3 hours, and persistence of good capillary refill was used as an endpoint for high-dose pulsed hyaluronidase treatment. Shortly thereafter, the patient was discharged with oral antibiotic cover.

On the third post-injection day, tiny skin blisters started appearing on the patient's nose tip and along the nose dorsum, with some skin exfoliation and peeling (Fig. 2). Since the blisters appeared to be drying out, and adequate tissue reperfusion continued, further hyaluronidase treatment was not required. The patient reported that the pain was much reduced, and the nasal tip much less swollen. Shortly thereafter, we made the decision to send the patient for hyperbaric oxygen therapy

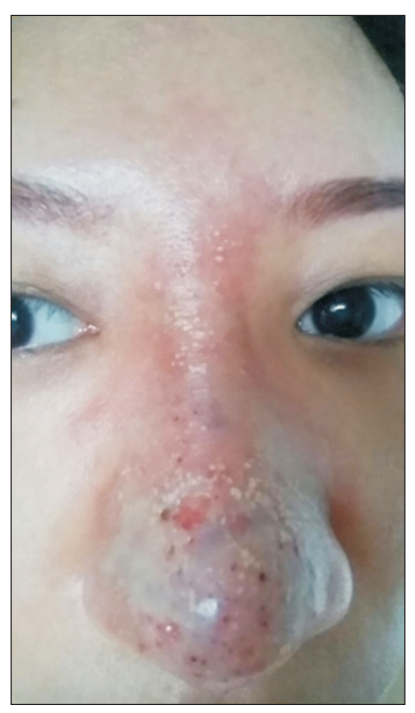

Fig. 2. Third day post filler injection, showing skin exfoliation, and noticeable swelling of full thickness tissue, mainly at the nose tip area. Some blistering is beginning to appear. 


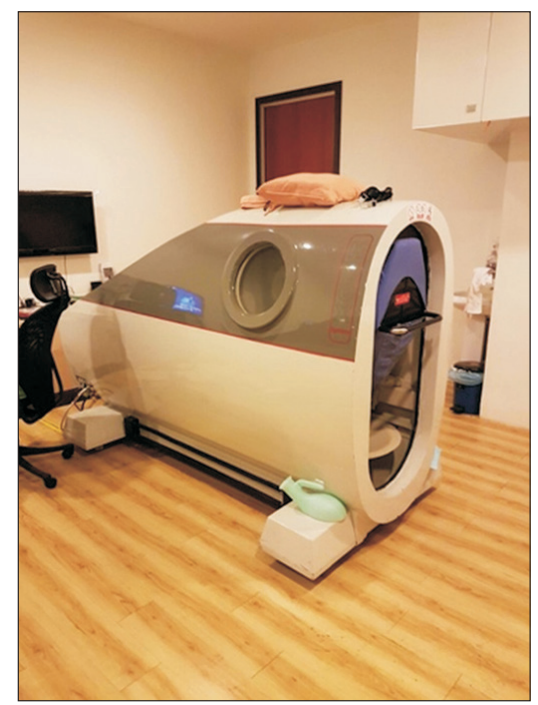

Fig. 3. Hyperbaric oxygen chamber.

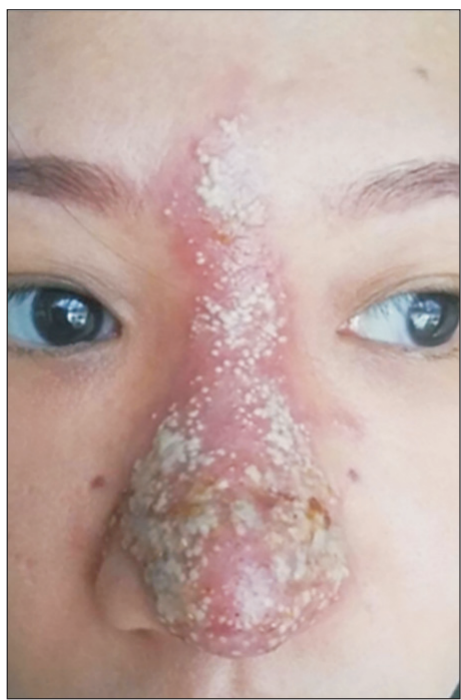

Fig. 4. Fourth day post filler injection, and after 1st session of hyperbaric oxygen therapy. The area of demarcation and blistering became more obvious, and the area of damage extended up to the glabellar region. The nasal tip became much less swollen.

(НBOT) in a hyperbaric oxygen chamber as shown in Fig. 3, with 30-minute treatment sessions, at 2 absolute atmospheres (ATA). After one hyperbaric oxygen treatment (Fig. 4), the area of demarcation and blistering became more obvious, and the nasal tip became much less swollen. At this stage, the patient reported only minimal pain and felt comfortable. Previous reports have shown positive results with HBOT for wound revascularization and skin grafting.

A total of four HBOT sessions at 2 ATA were performed in this case, one daily 30-minute treatment, 4 days consecutively.

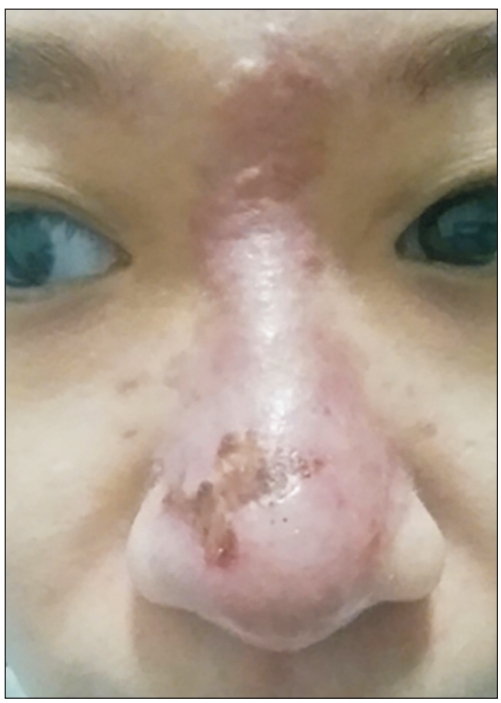

Fig. 5. Seventh day post injection, completing four sessions of hyperbaric oxygen therapy. The wound had completely dried with residual regenerating fresh tissue and almost complete skin peeling.

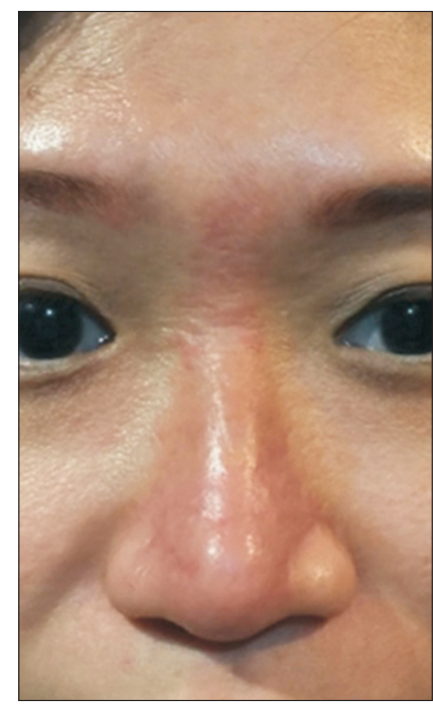

Fig. 6. Thirteen days after filler injection, the blisters had healed with minimal residual scarring.

By the end of the 3rd hyperbaric oxygen treatment, the patient no longer felt any pain or discomfort, blistering had ceased, wounds were drying, and full thickness tissue regeneration was underway. On the seventh day after filler injection, the patient felt some itching, wound was completely dry, and fresh tissue was regenerating with almost complete peeling of the superficial skin (Fig. 5).

Two weeks post filler injection, the blisters had healed with minimal residual scarring, but the patient still felt some itching (Fig. 6). After the third month and onwards, the nose had com- 
pletely healed with no residual scarring, demonstrating that the treatment was effective in reversing ischemic skin changes and successfully precluding any need for surgical intervention or any further scar management treatment (Fig. 7).

\section{Signs and symptoms}

Pain: severe pain is an important identifying feature of intraarterial filler injection, however it may not be immediately perceived by the patient due to the local anesthetic typically mixed and injected with most fillers. If a patient complains of severe pain during treatment, or during the subsequent hours after treatment, the practitioner should be alerted to the risk of

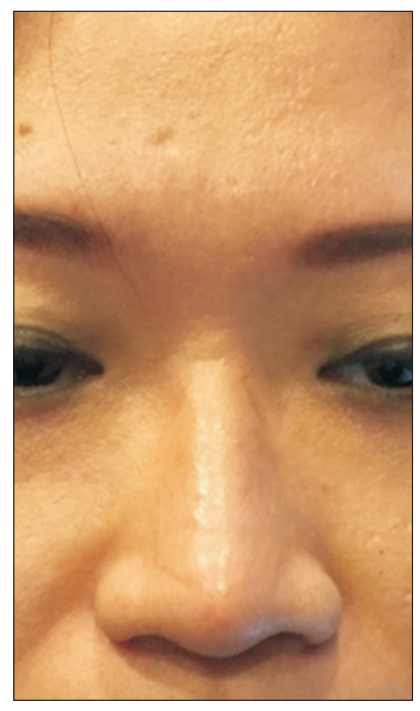

Fig. 7. Three months after filler injection, the nose healed completely with no residual scarring. impending necrosis and an urgent review is warranted.

Prolonged blanching: when the vasculature is affected, CRT is slow, and the area will often initially look very pale, white, or dusky due to blood supply reduction, and this color will remain after removal of the needle. The blanching pattern is often described as reticulated or irregular, following the same path as the restricted blood supply.

Dusky, purple discoloration: This discoloration typically occurs several hours after treatment, when tissue death has already occurred.

Coolness: when blood supply is affected preventing tissue perfusion, skin temperature will be reduced. The change in temperature is usually apparent a few hours after injection.

\section{Discussion}

Intra-arterial injection may be identified by blanching followed by a livedo pattern, coupled with severe pain. In most studies, blanching has been described as transient or lasting only a few seconds, but in some cases may last for hours.

The main purpose of this case report on intravascular filler injection was to capture knowledge that will help other injector physicians learn and recognize early signs of ischemic skin necrosis, and promptly begin management. In the event of arterial/venous occlusion and impending necrosis, the goal is to quickly promote increased blood flow to the affected area, so treatment may commence without delay (Table 1).

In our case study, most likely nasal arterial occlusion occurred due to several strong indicators, including blanching, delayed capillary reperfusion of the affected tissue, and a well-

Table 1. Management of impending necrosis: treatment protocol and expert recommendations.

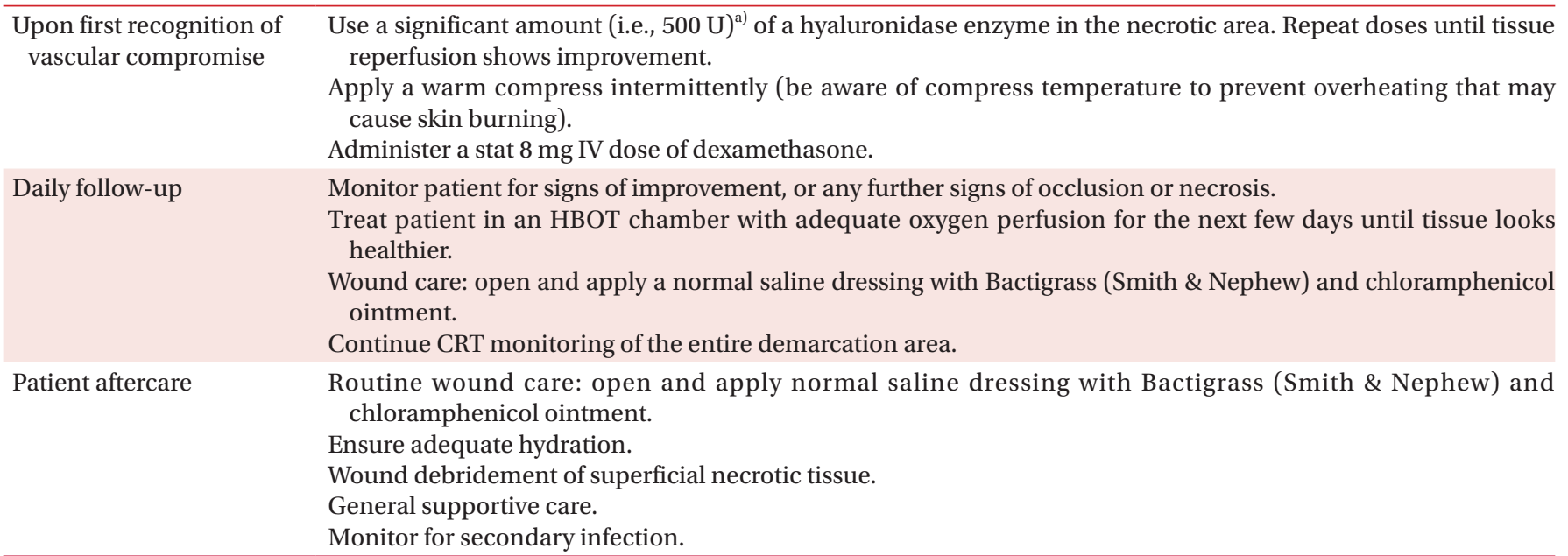

IV, intravenous; HBOT, hyperbaric oxygen therapy; CRT, capillary refill time.

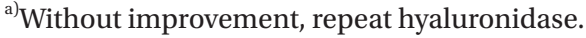




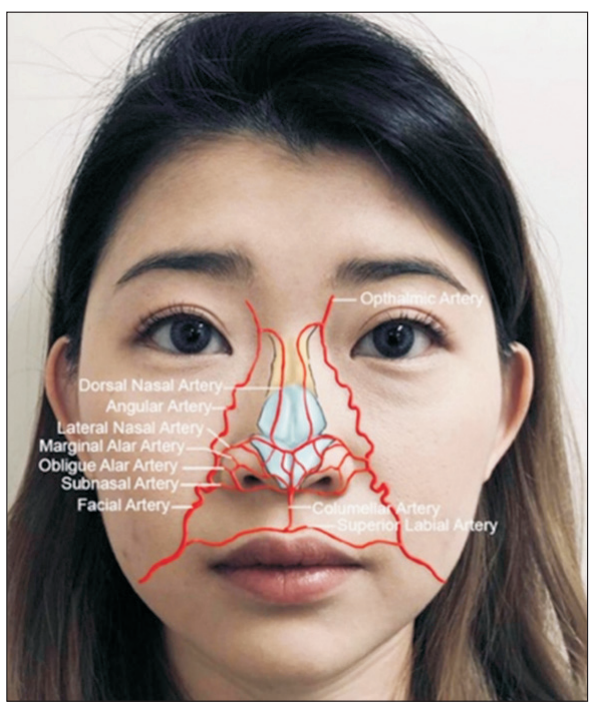

Fig. 8. Anatomical arterial supplies and anastomosis of multiple end arteries to the nose tip, alar nose, columella of nose, and nasal ridge.

defined area of demarcation of impending necrosis a few days following the injections (Fig. 8).

Initial identification of the arterial territory potentially involved enables treatment of the entire affected area with hyaluronidase. In this case, the dorsal nasal artery and lateral nasal artery were involved affecting the nasal tip and nasal ridge skin up to the glabellar area.

It is essential for every aesthetic physician with an injectables practice to maintain stores of hyaluronidase for management of intra-vascular HA filler-related cutaneous complications. The estimated hyaluronidase dose varies depending on the thickness and number of affected areas.

In our case study, a higher hyaluronidase dose was required since two areas were involved, primarily the nose, and also the nasal ridge up to the glabellar area. The ideal time to begin treatment after an intra-arterial filler injection is immediately after diagnosis, but certainly not later than 48 hours, to avoid skin necrosis, permanent scarring, and a need for surgical reconstruction. Other reports have estimated treatment with 500 units of high-dose pulsed hyaluronidase for a vascular territory similar to the present case. Therefore, we adhered to that proto$\operatorname{col}[9]$.

The application of HBOT is considered routine in cases of acute peripheral arterial ischemia and impending skin necrosis due to dermal filler injection. In the United States, the Food and Drug Administration approved HBOT for necrotizing soft tissue infections and chronic, non-healing wounds. Although the utility of HBOT in case of vascular compromise due to cosmetic dermal injection has not been explored, it may improve aesthetic outcomes with only a few sessions during the initial critical period, with minimal cost.

HBOT is thought to be efficacious in these situations due to mechanisms such as oxygenation of ischemic tissues, reduction of edema, amelioration of ischemic/reperfusion injury, promotion of angiogenesis, and collagen maturation [10].

\section{Conclusion}

Although most studies suggest hyaluronidase injection, there is no unanimity on dosage, and the necessary interval between 2 doses. Hyaluronidase may be injected on an hourly basis until a treatment endpoint showing skin reperfusion and blanching/ livedo correction. As the hyaluronidase concentration in the affected tissue decreases over time due to degradation, dilution with extra-cellular fluid, and diffusion into surrounding tissue, replenishment at regular intervals is necessary to maintain a high concentration in the affected tissue. This new high-dose pulsed hyaluronidase treatment modality operates on the principle that an adequate amount of hyaluronidase at high concentration levels and a sufficiently long duration is required to dissolve HA material present in the vascular territory. This approach, coupled with HBOT, delivers necessary oxygen for wound healing to the injured tissues.

\section{Conflicts of interest}

The author has nothing to disclose.

\section{References}

1. Souza Felix Bravo B, Klotz De Almeida Balassiano L, Roos Mariano Da Rocha C, Barbosa De Sousa Padilha C, Martinezt Torrado C, Teixeira Da Silva R, et al. Delayed-type necrosis after soft-tissue augmentation with hyaluronic acid. J Clin Aesthet Dermatol 2015;8:42-7.

2. Cohen JL, Biesman BS, Dayan SH, DeLorenzi C, Lambros VS, Nestor MS, et al. Treatment of hyaluronic acid filler-induced impending necrosis with hyaluronidase: consensus recommendations. Aesthet Surg J 2015;35:844-9.

3. King M. This month's guideline: management of necrosis. J Clin Aesthet Dermatol 2018;11:E53-7.

4. Woodward J, Khan T, Martin J. Facial filler complications. Facial Plast Surg Clin North Am 2015;23:447-58.

5. Inoue K, Sato K, Matsumoto D, Gonda K, Yoshimura K. Arte- 
rial embolization and skin necrosis of the nasal ala following injection of dermal fillers. Plast Reconstr Surg 2008;121:1278 e.

6. Loh KTD, Phoon YS, Phua V, Kapoor KM. Successfully managing impending skin necrosis following hyaluronic acid filler injection, using high-dose pulsed hyaluronidase. Plast Reconstr Surg Glob Open 2018;6:e1639.

7. Sclafani AP, Fagien S. Treatment of injectable soft tissue filler complications. Dermatol Surg 2009;35 Suppl 2:1672-80.
8. Ozturk CN, Li Y, Tung R, Parker L, Piliang MP, Zins JE. Complications following injection of soft-tissue fillers. Aesthet Surg J 2013;33:862-77.

9. Hong JY, Seok J, Ahn GR, Jang YJ, Li K, Kim BJ. Impending skin necrosis after dermal filler injection: a "golden time" for first-aid intervention. Dermatol Ther 2017;30:e12440.

10. Henderson R, Reilly DA, Cooper JS. Hyperbaric oxygen for ischemia due to injection of cosmetic fillers: case report and issues. Plast Reconstr Surg Glob Open 2018;6:e1618. 